\title{
Air Oxidation of Sulfur Mustard Gas Simulant Using a Pyrene- Based Metal-Organic Framework Photocatalyst
}

Ghada Ayoub, ${ }^{\mathrm{a}, \mathrm{b}}$ Mihails Arhangelskis, ${ }^{\mathrm{a}}$ Xuan Zhang, ${ }^{\mathrm{b}}$ Florencia Son, ${ }^{\mathrm{b}}$ Timur Islamoglu, ${ }^{\mathrm{b}}$ Tomislav Friščić, ${ }^{* a}$ and Omar K. Farha*b,c

Abstract We demonstrate a microporous metal-organic framework NU-400 based on a 2,7disubstituted pyrene linker as a highly efficient photosensitizer for generating singlet oxygen and subsequent oxidative degradation of chemical warfare agents (CWAs). The high activity of NU400 permits photocatalytic conversion of the 2-chloroethyl ethyl sulfide (CEES) mustard gas simulant into a benign sulfoxide derivative, in air, with less than 15 minutes half-life. This is a considerable improvement to NU-1000, based on a 1,3,6,8-tetrasubstituted pyrene unit, demonstrating how variation of the substitution pattern of a metal-organic framework linker permits modification of its photoactive behavior.

Sulfur mustard gas also known as mustard gas, HD, or Yperite belongs to a class of chemical warfare agents (CWAs) known as vesicants, which have detrimental effects on humans, including the blistering of skin upon contact. ${ }^{1}$ Even at a low dosage, ${ }^{2}$ this chemical can be fatal. Although in 1993 at the Chemical Weapon Convention (CWC) 192 nations signed an agreement to outlaw the production, stockpiling, and deployment of chemical weapons, sulfur mustard gas has continuously been used against civilians and soldiers over the past several decades, ${ }^{3}$ including as recently as 2018 in Syria. ${ }^{4,5}$ Therefore, it is imperative to design and develop novel methods for the detoxification of sulfur mustard gas in stockpiles as well as in the battle field.

There are several routes for the detoxification of sulfur mustard gas, including: 1) hydrolysis,6,7 2) oxidation, and 3) dehydrohalogenation ${ }^{8-10}$ (scheme 1). The hydrolysis route is limited to small scales because of the hydrophobicity of sulfur mustard gas. The mechanism of degradation by dehydrohalogenation mechanism is still poorly understood and not efficient enough for real-world applications. So far, the oxidative degradation ${ }^{11-17}$ route has been shown to 
be the most promising but relies on the use of oxidants highly selective for the formation sulfoxides, as further oxidation to a sulfone leads to a product of toxicity comparable to sulfur mustard gas. ${ }^{18}$

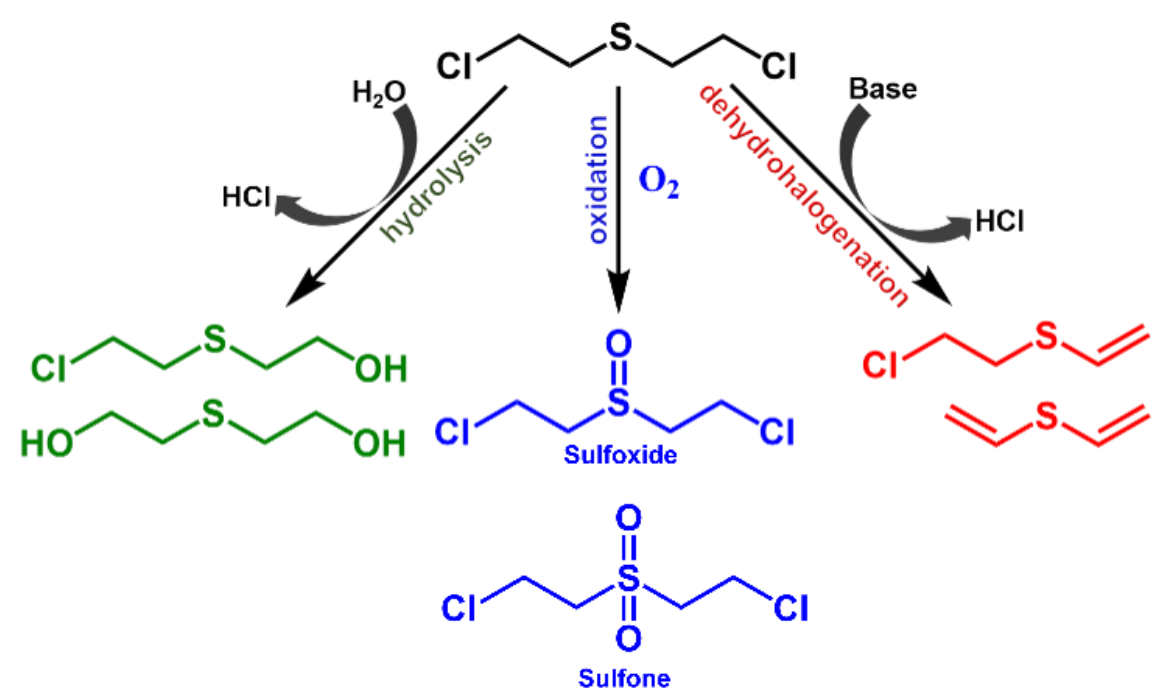

Scheme 1. Detoxification routes of sulfur mustard gas: hydrolysis (green); oxidation to sulfoxide (blue) and dehydrohalogenation (red).

While oxidative detoxification of sulfur mustard gas has traditionally relied on bleaching powders, such reagents come with significant drawbacks, including corrosiveness and loss of activity over time. ${ }^{19,20}$ Ideal candidates for the oxidation of mustard gas should be mild oxidants that will selectively produce the partially oxidized sulfoxide. In that context, a promising approach is the use of singlet oxygen $\left({ }^{1} \mathrm{O}_{2}\right)$ generated by photosensitization of ground-state molecular oxygen $\left({ }^{3} \mathrm{O}_{2}\right)$ via an electronically excited chromophore. The chromophores can be compounds with significant quantum yields and whose absorption wavelengths span the ultraviolet-visible spectrum. Moreover, singlet oxygen could be generated from homogenous ${ }^{21}$ or heterogeneous catalysts, the latter of which will allow for the ease of separation at the end of the reaction, recyclability of the catalyst, wide selection of solvent choices since solubility of the chromophore does not need to be considered, and lower probability of photobleaching. ${ }^{22,23}$

Metal-organic framework (MOFs), porous crystalline materials comprised of metal nodes and organic linkers, have attracted tremendous attention in heterogeneous catalysis due to their structural and chemical tunability. ${ }^{24-34}$ In that context, zirconium-based MOFs have demonstrated 
particularly high stability under a range of conditions, enabling their application for efficient, rapid hydrolytic or oxidative degradation of nerve gas agents. ${ }^{35-41}$

Here, we prepared NU-400, ${ }^{42}$ a zirconium-based MOF based on a judiciously chosen pyrene-based linker and utilized it as a photosensitizer for the efficient production of ${ }^{1} \mathrm{O}_{2}$ and hence photocatalytic conversion of the sulfur mustard simulant 2-chloroethyl ethyl sulfide (CEES) into a benign sulfoxide product, using ambient air as the oxygen source. We selected a $\mathrm{Zr}_{6}$-based MOF because of its outstanding stability under a wide range of thermal and chemical conditions. As pyrene has been known as an efficient photosensitizer that is capable of producing singlet oxygen upon exposure to UV-light, ${ }^{43,44}$ we anticipated that a MOF with isolated pyrene linkers would be good a candidate catalyst for the photocatalytic oxidation of sulfur mustard.

The NU-400 material (Fig. 1) was synthesized from the 2,7-pyrenedicarboxylic acid (Py-DCA) linker, $\mathrm{ZrCl}_{4}$ metal salt, and acetic acid as a modulator, in DMF at $120{ }^{\circ} \mathrm{C}$ (see S.3 for synthetic details). Different from the reported synthesis of 2,7-pyrene dicarboxylic acid linker, ${ }^{45}$ which required an organolithium reagent, a more benign Pd-catalyzed carbonylation reaction was utilized with 2,7-dibromopyrene as the starting material. ${ }^{46}$ Powder X-ray diffraction (PXRD) analysis of the as-synthesized materials revealed that $\mathrm{NU}-400$ is isostructural to the related UiO-67 framework based on 4,4'-biphenyldicarboxylate linkers. Subsequently, the structure of NU-400 (see S.2.1, Table S1 for crystal structure details) was established from PXRD data, by Rietveld refinement (Fig. S1) of a model generated from UiO-67 (CCDC code WIZMAV03). The morphology of the materials was confirmed by scanning electron microscopy (SEM) images, which revealed that bulk NU-400 material consists of octahedral crystals with sizes ranging from 1 to 5 microns (Fig. S3). The microporous nature of NU- 400 was established by $\mathrm{N}_{2}$ sorption measurements at $77 \mathrm{~K}$, which revealed a Brunauer-Emmet-Teller (BET) surface area of $1325 \mathrm{~m} 2 / \mathrm{g}$ (Fig. S4). ${ }^{45}$ The pore size analysis using DFT model revealed pores of approximately $11 \AA$, which is suitable for diffusion of CEES molecules into the pores of NU-400. 
a)

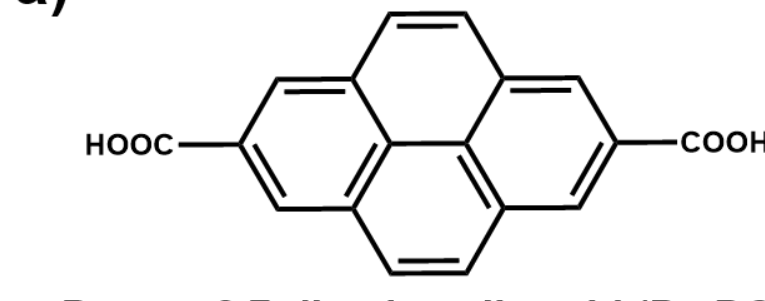

Pyrene-2,7-dicarboxylic acid (Py-DCA)

b)

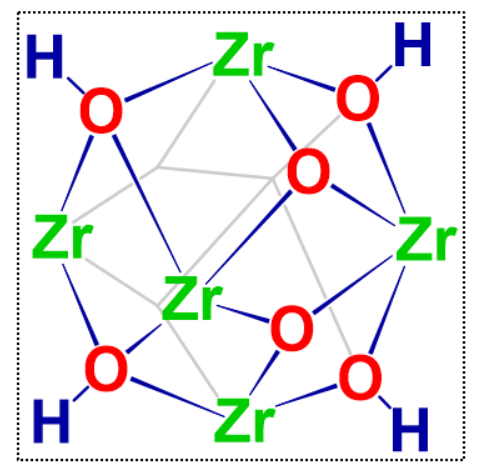

Ideal node c)

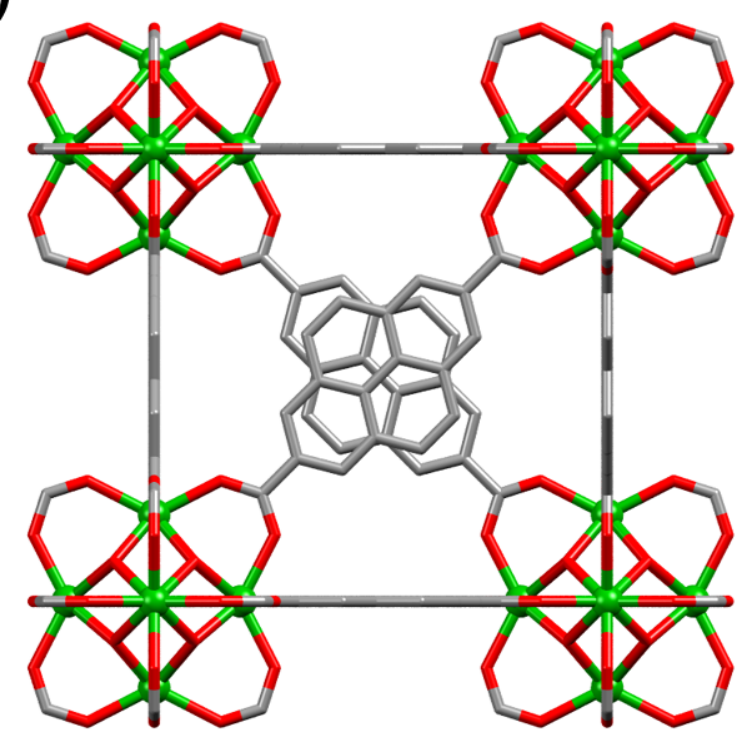

NU-400

Figure 1. NU-400 constituents: a) the pyrene-based linker, 2,7-pyrene dicarboxylic acid and b) $\mathrm{Zr}_{6}$ metal node. c) Fragment of the crystal structure of NU-400, established from PXRD data. Hydrogens atoms and disorder of pyrene groups are not shown for clarity Zr: green, O: red, and C: grey.

Solid-state UV-Vis spectrum of NU-400 reveals that the strongest absorption bands lie below 400 nm (see Fig. S5), leading us to use two commercially available ultraviolet light emitting diodes (UV-LEDs) with $\lambda \max =390-400 \mathrm{~nm}$ as a means to excite the MOF for ${ }^{1} \mathrm{O}_{2}$ production.

As our first entry into investigating the efficiency of NU-400 as a photosensitizer for singlet oxygen generation, we used $1 \mathrm{~mol} \%$ ( $2 \mu \mathrm{mol}, 4.75 \mathrm{mg}$ ) of the MOF under oxygen saturation conditions and UV-irradiation (see S.4 for detailed procedure). Aliquots were taken at various time points, filtered using syringe filters and, after dilution with dichloromethane, analyzed by GC-FID to monitor the reaction kinetics. Oxidation products were analyzed by NMR spectroscopy using deuterated methanol as a solvent. Under these conditions, reaction monitoring revealed that complete and selective conversion of CEES into CEESO was achieved over a period of 50 minutes, with a half-life of 10.2 minutes (Fig. 2). During control experiments designed to evaluate the significance of each parameter in the process of ${ }^{1} \mathrm{O}_{2}$ production, we unexpectedly found that 
conducting the photocatalytic oxidation under the same conditions of irradiation, and the same MOF content, but in the absence of $\mathrm{O}_{2}$ saturation step, also led to complete conversion of CEES into the sulfoxide. Specifically, under such conditions the complete conversion of CEES was observed after 2 hours, with a half-life of 13.5 minutes (Fig. 2). Achieving complete oxidation of CEES without the $\mathrm{O}_{2}$ saturation represents a milestone for the potential deployment of MOFs as an active detoxification catalyst and, consequently, we focused on detailed exploration of the activity of NU-400 in air, without oxygen purging.

Given that NU-400 is an active photocatalyst, where the pyrene-based linkers are expected to play the role of photosensitizers responsible for singlet oxygen production under UV-irradiation, several control studies were performed to firmly establish the role of the linker. We explored the ability of pure linker precursor to act as the photosensitizer by performing the oxidation in the presence of $1 \mathrm{~mol} \%$ Py-DCA $(3.4 \mathrm{mg}, 11.5 \mu \mathrm{mol})$ under air and in the presence of UV-light, leading to a $75 \%$ conversion of CEES to CEESO after 2 hours (Fig. 2). This observation implies that incorporation into the MOF structure enhanced the catalytic activity of Py-DCA, most likely due to the heterogeneous nature of the MOF, which assembles the pyrene linkers periodically within a robust three-dimensional framework, preventing their deactivation through aggregation. In all cases the ${ }^{1} \mathrm{O}_{2}$ acted as a highly selective oxidant for the formation of the target sulfoxide species, as no overoxidation to form a more toxic sulfone analogue was observed (Fig. S6). Finally, we explored the possibility of reaction occurring in the absence of UV-light. Under these conditions, in the presence of $1 \mathrm{~mol} \%$ (12 $\mu \mathrm{mol}, 3.4 \mathrm{mg})$ of the MOF, no conversion of CEES conversion was detected (Fig. 2), confirming the role of NU-400 as a photocatalyst. 
a)

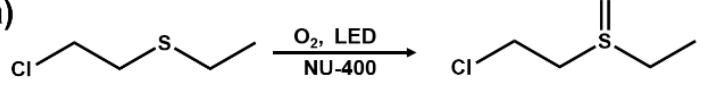

b)

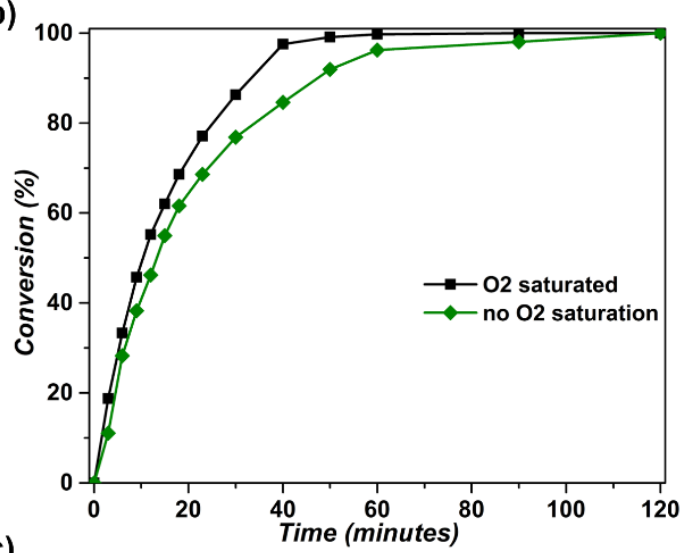

c)

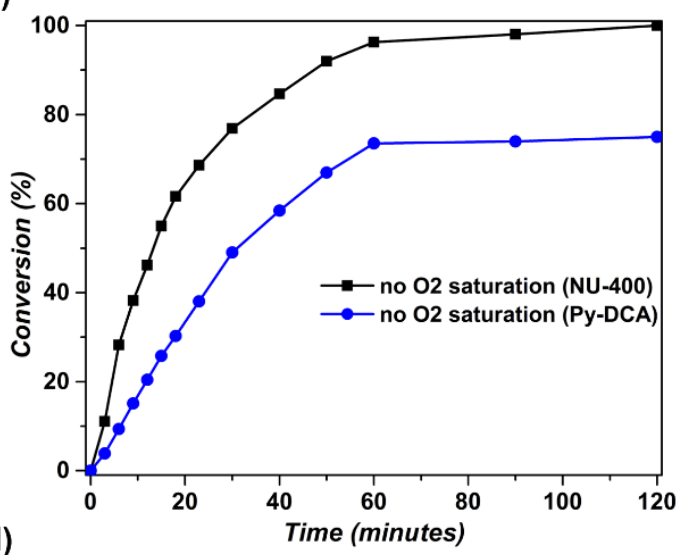

d)

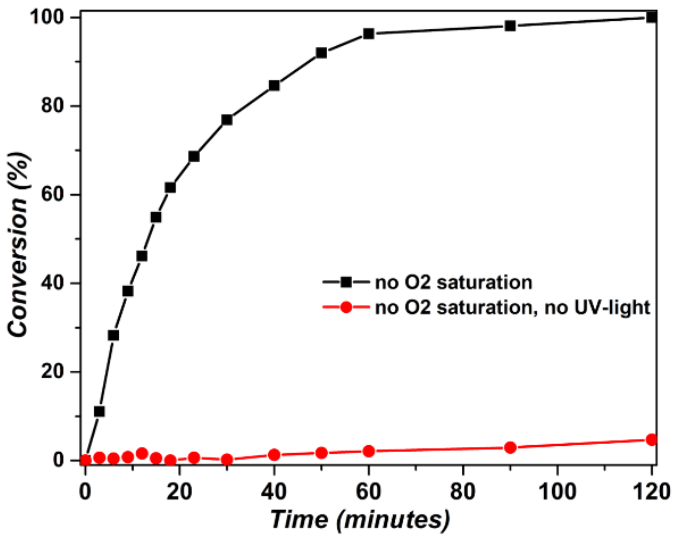

Figure 2. Reaction of CEES conversion to CEESO under different conditions: (a) reaction scheme; (b) in the presence of NU-400 with $\mathrm{O}_{2}$ saturation and under air; c) in the presence of Py-DCA with $\mathrm{O}_{2}$ saturation and under air, and d) in the presence or absence of UV-light, under air. One mol\% (based on Py-DCA) catalyst loading was used for all reactions.

The photocatalytic activity of NU-400 in air, with-out oxygen presaturation, is significantly higher compared to the previously explored mesoporous NU-1000 MOF, which is based on a different, 
tetratopic pyrene-based linker 4,4',4', 4','-(pyrene-1,3,6,8-tetrayl)tetrabenzoate (H4TBApy). Using $1 \mathrm{~mol} \%(5.2 \mathrm{mg}) \mathrm{NU}-1000$ as a photocatalyst enabled the full conversion of CEES into CEESO with a half-life of only 6.2 minutes und ${ }_{2}$ er conditions of $\mathrm{O}_{2}$ saturation. However, the process was significantly slower, with a half-life of 24.5 minutes (Fig. 3), when the reaction vessel was not saturated with oxygen. The superior performance of NU-400 (half-life of 13.5 minutes) under air can be attributed to the higher density of pyrene linkers in NU-400 $\left(0.101 \mathrm{~g} / \mathrm{cm}^{3}\right)$ compared to NU-1000 $\left(0.0506 \mathrm{~g} / \mathrm{cm}^{3}\right)$ which is responsible for ${ }^{1} \mathrm{O}_{2}$ generation.

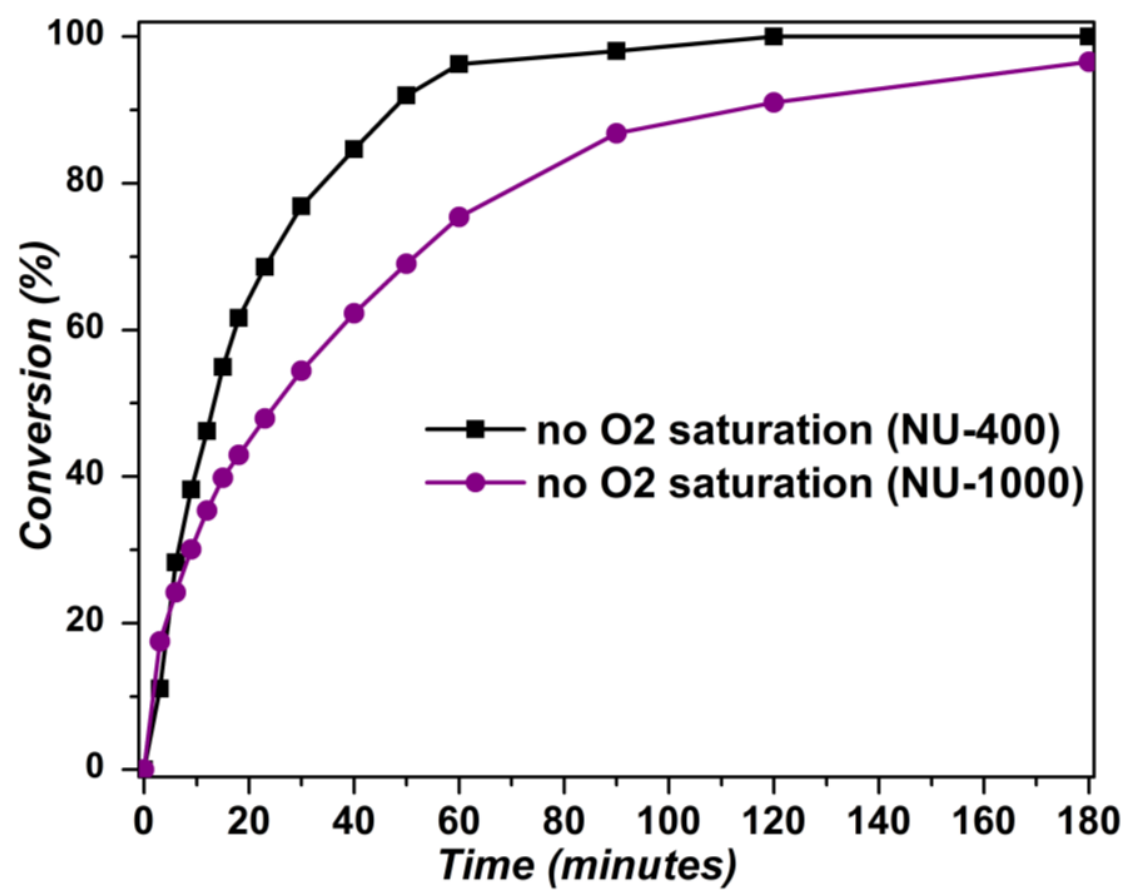

Figure 3. Selective oxidation of CEES to CEESO using $1 \mathrm{~mol} \%$ catalyst of NU-400 (black) versus NU-1000 (purple).

Finally, we investigated to recyclability of NU-400 by adding multiple injections of CEES $(0.2$ $\mu \mathrm{mol})$ into the microwave vial after one cycle of full conversion of CEES to CEESO. As the reaction was carried out using the oxygen available in atmosphere, without any additional $\mathrm{O}_{2}$ purging, opening the microwave vial upon the addition of CEES ensured the presence of fresh air needed for the reaction. This recyclability test was repeated three times, and the reaction progress was monitored using GC-FID in order to calculate the conversion of the reaction after each injection (Fig. 4). 


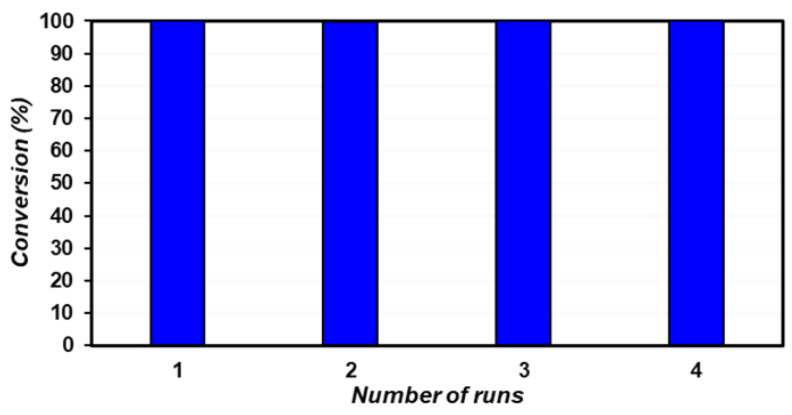

Figure 4. The reusability of the catalyst NU-400 MOF over four successive injections of CEES $(0.2 \mathrm{mmol})$ into the same reaction vial.

The photocatalytic oxidation reaction takes place without noticeable degradation of NU-400, as evidenced by PXRD analysis following four cycles of CEES to CEESO oxidation, which reveals a high degree of crystallinity (see Fig. S7). At the same time, no overoxidation of CEES to 2chloroethyl ethyl sulfone $\left(\mathrm{CEESO}_{2}\right)$ was observed, as demonstrated by ${ }^{1} \mathrm{H}$ NMR spectroscopy (Fig. S6).

In summary, we demonstrated NU-400, a microporous MOF based on a 2,7-dicarboxylate pyrene linker as a highly effective platform for singlet oxygen production and photocatalytic degradation of mustard gas simulant. In contrast to previously reported NU-1000, based on a 1,3,6,8-tetrasubstituted pyrene unit, which required saturation with oxygen to achieve effective high singlet oxygen production, the herein reported NU-400 is effective without oxygen saturation. The photocatalytic activity of NU-400 enabled singlet oxygen-induced conversion of CEES to CEESO with a half-life of 13.5 minutes under air, a milestone in the development of MOFs as new, highly efficient catalysts for mustard gas degradation.

\section{Associated content}

\section{Supporting Information}

The Supporting Information is available free of charge on the

ACS Publications website at DOI: $x x x x x x x x x x x x x x x x x$

Specifics on materials used, MOF synthesis, details of characterization, instrumentation.

\section{Author Information}

\section{Corresponding Author}

*Prof. Omar K. Farha, Department of Chemistry, Northwestern University, 2145 Sheridan Rd, Evanston, IL 6o2o8, USA. E-mail: o-farha@northwestern.edu

${ }^{*}$ Prof. Tomislav Friščić, Department of Chemistry, McGill University, 8o1 Sherbrooke St. W. H3A oB8 Montreal, Canada.

E-mail: tomislav.friscic@mcgill.ca

Notes

The authors declare no competing financial interests. 


\section{ACKNOWLEDGMENT}

O.K.F. gratefully acknowledges support from the Defense Threat Reduction Agency (HDTRA1-18-1-ooo3). T.F. acknowledges financial support of the National Science and Engineering Research Council (NSERC) Discovery Grant (RGPIN-2017-06467), NSERC E.W.R. Steacie Memorial Fellowship (SMFSU 507347-17) and McGill University Graduate Student Mobility Award (for GA), and Northwestern University.

\section{REFERENCES}

1. Fitzgerald, G. J. Am. J. Public Health 2008, 98, 611-625.

2. Sidell, F.R.; Borak, J. Chemical warfare agents: II. Nerve agents. Ann. Emerg. Med. 1992, 21, 865-871.

3. Organisation for the Prohibition of Chemical Weapons. Chemical Weapons Convention. https://www.opcw.org/chemical-weapons-convention/ (accessed Aug 19, 2016).

4. Hess, G. UN says Syria and Islamic State used chemical weapons, Chemical and Engineering News, August 2016.

5. Fassihi, F. U. N. Report Finds Chemical Weapons Used by Syrian Regime, Islamic State, The Wall Street Journal, August 2016.

6. Wilderer, P. A.; Irvine, R. L.; Doellerer, J.; Irvine, D. A.; Earley, J. P.; Cassidy, D. P.; Harvey, S. P. Water Sci. Technol. 1997, 35, 67-74.

7. $\quad$ Wang, Q.-Q.; Begum, R. A.; Day, V. W.; Bowman-James, K. Org. Biomol. Chem. 2012, 10, 8786-8793.

8. Wagner, G. W.; Bartram, P. W.; Koper, O.; Klabunde, K. J. J. Phys. Chem. B 1999, 103, 3225-3228

9. Wagner, G. W.; Koper, O. B.; Lucas, E.; Decker, S.; Klabunde, K. J. J. Phys. Chem. B 200o, 104, 5118-5123.

10. Horcajada, P.; Surble, S.; Serre, C.; Hong, D.-Y.; Seo, Y.-K.; Chang, J.-S.; Greneche, J.-M.; Margiolaki, I.; Ferey, G. Chem. Commun. 2007, 2820-2822.

11. Popiel, S.; Nawała, J. Enzyme Microb. Technol. 2013, 53, 295-301.

12. Price, C. C.; Bullitt, O. H. J. Org. Chem. 1947, 12, 238-248.

13. Gall, D.; Faraj, M.; Hill, C. L. Inorg. Chem. 1994, 33, 5015-5021.

14. Richardson, D. E.; Yao, H.; Frank, K. M.; Bennett, D. A. J. Am. Chem. Soc. 20oo, 122, 1729-1739.

15. Boring, E.; Geletii, Y. V.; Hill, C. L. J. Am. Chem. Soc. 20o1, 123, 1625-1635.

16. Ringenbach, C. R.; Livingston, S. R.; Kumar, D.; Landry, C. C. Chem. Mater. 2005, 17, 5580-5586.

17. Wagner, G. W.; Yang, Y.-C. Ind. Eng. Chem. Res. 2002, 41, 1925-1928.

18. Hirade, J.; Ninomiya, A. J. Biochem. 1950, 37, 19-34.

19. Yang, Y. C.; Baker, J. A.; Ward, J. R. Chem. Rev. 1992, 92, 1729-1743

20. Jang, Y. J.; Kim, K.; Tsay, O. G.; Atwood, D. A.; Churchill, D. G. Chem. Rev. 2015, 115, PR1-PR76.

21. DeRosa, M.C. and Crutchley, R.J. Coord. Chem. Rev. 2oo2, 233, 351-371.

22. Griesbeck, A.G.; El-Idreesy, T.T.; Bartoschek, A. Adv. Synth. Catal. 2004, 346, 245-251.

23. Roman, E. San. Singlet Oxygen: Applications in Biosciences and Nanosciences, The Royal Society of Chemistry, 2016, 1, 183-208.

24. Li, H.; Eddaoudi, M.; O'Keeffe, M.; Yaghi, O. M. Nature 1999, 402, 276-279.

25. Férey, G. Chem. Soc. Rev., 2008, 37, 191-214.

26. Cooper, A. I.; Rosseinsky, M. J. Nat. Chem. 2oo9, 1, 26-27.

27. Shimizu, G. K. H.; Vaidhyanathan, R.; Taylor, J. M. Chem. Soc. Rev. 20o9, 38, 1430-1449

28. Zhou, H.-C.; Long, J. R.; Yaghi, O. M. Chem. Rev. 2012, 112, 673-674

29. Chen, Z., Hanna, S.L., Redfern, L.R., Alezi, D., Islamoglu, T. and Farha, O.K. Coord. Chem. Rev. 2019, 386, 32-49.

30. Denny, M. S., Jr.; Moreton, J. C.; Benz, L.; Cohen, S. M. Nat. Rev. Mater. 2016, 1, 16078-16085.

31. Smaldone, R.A.; Forgan, R.S.; Furukawa, H.; Gassensmith, J.J.; Slawin, A.M.; Yaghi, O.M.; Stoddart, J.F. Angew. Chem. Int. Ed. 2010, 49, 8630-8634.

32. Campbell, M. G.; Liu, S. F.; Swager, T. M.; Dincă, M. J. Am. Chem. Soc. 2015, 137, 13780-13783

33. Orellana-Tavra, C.; Baxter, E. F.; Tian, T.; Bennett, T. D.; Slater, N. K. H.; Cheetham, A. K.; Fairen-Jimenez, D. Chem. Commun. 2015, 51, 13878-13881

34. Lee, J.; Farha, O. K.; Roberts, J.; Scheidt, K. A.; Nguyen, S. T.; Hupp, J. T. Chem. Soc. Rev. 2009, 38, $1450-1459$.

35. Ayoub, G.; Islamoglu, T.; Goswami, S., Friščić, T. and Farha, O.K. ACS Appl. Mater. Interfaces 2019, 11, 1578815794 . 
36. Howarth, A.J.; Liu, Y.; Li, P.; Li, Z.; Wang, T.C.; Hupp, J.T.; Farha, O.K. Nat. Rev. Mater. 2016, 1, 15018.

37. Feng, D.; Gu, Z.Y.; Li, J.R.; Jiang, H.L.; Wei, Z.; Zhou, H.C. Angew. Chem. Int. Ed. 2012, 51, 10307-10310.

38. Liu, Y.; Howarth, A.J.; Vermeulen, N.A., Moon, S.Y., Hupp, J.T. and Farha, O.K. Coord. Chem. Rev. 2017, 346, 101111.

39. Buru, C.T.; Majewski, M.B.; Howarth, A.J.; Lavroff, R.H.; Kung, C.W.; Peters, A.W.; Goswami, S.; Farha, O.K. ACS Appl. Mater. Interfaces 2018, 10, 23802-23806.

40. Gil-San-Millan, R.; López-Maya, E.; Hall, M.; Padial, N.M.; Peterson, G.W.; DeCoste, J.B.; Rodríguez-Albelo, L.M.; Oltra, J.E.; Barea, E.; Navarro, J.A. ACS Appl. Mater. Interfaces 2017, 9, 23967-23973.

41. DeCoste, J.B.; Peterson, G.W. Chem. Rev. 2014, 114, 5695-5727.

42. We note that during the preparation of our manuscript, this MOF was reported by Lv, X-L. and coworkers. See Lv, X.L.; Yuan, S.; Xie, L.H.; Darke, H.F.; Chen, Y.; He, T.; Dong, C.; Wang, B.; Zhang, Y.Z.; Li, J.R.; Zhou, H.C. J. Am. Chem. Soc. 2019, 141, 2054-2060.

43. Liu, Y.; Buru, C. T.; Howarth, A. J.; Mahle, J. J.; Buchanan, J. H.; DeCoste, J. B.; Hupp, J. T.; Farha, O. K. J. Mater. Chem. A 2016, 4, 13809-13813.

44. Bobbitt, N.S.; Mendonca, M.L.; Howarth, A.J.; Islamoglu, T.; Hupp, J.T.; Farha, O.K.; Snurr, R.Q. Chem. Soc. Rev. 2017, 46, 3357-3385.

45. Lv, X.L.; Yuan, S.; Xie, L.H.; Darke, H.F.; Chen, Y.; He, T.; Dong, C.; Wang, B.; Zhang, Y.Z.; Li, J.R.; Zhou, H.C. J. Am. Chem. Soc. 2019, 141, 2054-206o.

46. Zhang, X.; Huang, Z.; Ferrandon, M.; Yang, D.; Robison, L.; Li, P.; Wang, T. C.; Delferro, M.; Farha, O. K. Nat. Catal. 2018, 1, 356-362. 